\title{
The observed periods of Ap and Bp stars ${ }^{\star, \star \star}$
}

\author{
F.A. Catalano ${ }^{1}$ and P. Renson ${ }^{2}$ \\ ${ }^{1}$ Istituto di Astronomia dell'Università di Catania and C.N.R.-G.N.A. Unità di Ricerca di Catania, Città Universitaria, I-95125 \\ Catania, Italia \\ e-mail: fcatalano@alpha4.ct.astro.it \\ ${ }^{2}$ Institut d'Astrophysique, Université de Liège, Avenue de Cointe 5, B-4000 Liège, Belgium \\ e-mail: renson@astro.ulg.ac.be
}

Received April 1; accepted May 29, 1997

\begin{abstract}
A catalogue of all the periods up to now proposed for the variations of $\mathrm{CP} 2, \mathrm{CP} 3$, and $\mathrm{CP} 4$ stars is presented. The main identifiers (HD and HR), the proper name, the variable-star name, and the spectral type and peculiarity are given for each star as far as the coordinates at 2000.0 and the visual magnitude. The nature of the observed variations (light, spectrum, magnetic field, etc.) is presented in a codified way. The catalogue is arranged in three tables: the bulk of the data, i.e. those referring to CP2, CP3, and CP4 stars, are given in Table 1, while the data concerning He-strong stars are given in Table 2 and those for eclipsing or ellipsoidal variables are collected in Table 3. Notes are also provided at the end of each table, mainly about duplicities.

The catalogue contains data on $364 \mathrm{CP}$ stars and is updated to 1996, October 31.
\end{abstract}

Key words: catalogs — stars: chemically peculiar stars: variables: other.

\section{Introduction}

At present more than 6600 stars are known or suspected to be chemically peculiar in the range of spectral types from B1 through F5 (Renson et al. 1991). Among them about 3480 stars are classified as Am or CP1, according to Preston's (1974) scheme, and about 3160 stars are Ap and Bp stars (i.e. CP2, CP3, CP4, and He-strong).

A period has been proposed only for 342 stars belonging

\footnotetext{
Send offprint requests to: F.A. Catalano

* This research has made use of the SIMBAD database, operated at CDS, Strasbourg, France.

$\star \star$ Table only available in electronic form at CDS via anonymous ftp to cdsarc.u-strasbg.fr (130.79.128.5) or via http://cdsweb.u-strasbg.fr/Abstract.html
}

to the CP2, CP3, and CP4 groups of Preston's scheme, i.e. magnetic $\mathrm{Ap}$ ( $\mathrm{Si}, \mathrm{CrEu}, \ldots$ stars), $\mathrm{HgMn}$, and He-weak stars respectively. These stars are listed in Table 1 . Their variations are considered to be linked with nothing but axial rotation (via the oblique rotator model), i.e. the quoted periods are those of the star rotations. The very short periods, attributed to pulsations or oscillations (roAp stars) are not included.

It is generally assumed that the He-strong stars constitute the extention of the Ap phenomenon at higher temperatures. However it is often difficult to know if their variations are of the same nature (rotation) or originate from pulsations (pulsating He stars). Therefore $13 \mathrm{He}$ stars exhibiting a periodic variation probably originating from their rotation are listed in another table: Table 2. Some probably pulsating He stars with known periods (Rns 26510, 27090, 41964, 42920, 45230, 46600, 55266) are not included.

The 9 Ap stars for which the periodic luminosity variations are ascribed to a binary effect (eclipsing and/or ellipsoidal stars) are also gathered in a separate table: Table 3 . The distinction is not always obvious because their periods are of the same order as for other Ap stars.

\section{The catalogue}

The catalogue here presented is essentially the result of a large effort culminated in the publication of the General Catalogue of $A p$ and Am Stars (Renson et al. 1991). The stars are listed in order of increasing running number in that catalogue (henceforth Rns number).

In the various columns of all three tables we give:

1. the HD (or HDE) number, the HR (= BS) number, if any, and the running number (Rns) in the General Catalogue of Ap and Am stars (Renson et al. 1991);

2. another name of the star, the variable star name (if any), the right ascension at the epoch 2000.0. 
As far as the other name of the star is concerned, we give in order of preference: the greek letter (Bayer) or number (Flamsteed) in the constellation (for the austral constellations we give the Gould (G.) number), the GC number in the Boss (1937) catalogue, the Durchmusterung number (BD, CoD, or CpD). In case of a double star a capital letter $\mathrm{A}, \mathrm{B}, \ldots$ is appended to specify which component is considered; the capital letter is however omitted if the other component is far away or much weaker;

3 . the spectral type and peculiarity type, the visual magnitude $m_{V}$, and the declination at the epoch 2000.0. In the peculiarity type only the most abundant elements are considered. The abbreviation $\mathrm{He} w k$ means that the star belongs to the He weak subgroup. The values of the visual magnitude are generally quoted with two decimal digits; however such a precision is often illusive, especially when the luminosity variation is not very small. In case of double stars, when the angular separation of the components is only of the order of one or a few arcsec, the value found in the literature is the magnitude of the system as a whole; then a correction is applied in order to quote here the $m_{V}$ value of the Ap component alone (as it has been done by Renson in the Catalogue General des Etoiles Ap et $A m$, in which the $m_{V}$ values were however given with only one decimal digit);

4. the most probable period (in days), the type of variability, and $\mathrm{a} \star$ if there is a remark at the end of the catalogue.

The presence of a : after the period value indicates that it is approximate, a ? indicates that it is dubious. The type of variability is indicated according to the code: $\ell$ : luminosity and/or color, $m$ : magnetic field, $s$ : spectral line intensity, $v$ : wavelength displacements of the lines of an ion, interpreted as mean radial velocity variations for that ion;

5. source catalogues, with the codes: $0=$ main catalogue (Catalano \& Renson 1984), 1 = first supplement (Catalano \& Renson 1988), 2 = second supplement (Catalano et al. 1991), $3=$ third supplement (Catalano et al. 1993), $4=$ fourth supplement (Catalano \& Renson 1997), and blank = new reference or old reference only now added;

6. proposed values of the period (in days) with their accuracy, if available, and references. If more values are reported by an author we list them separated by the symbol / (which thus means: or). If the other values are less probable we put them in parentheses. The presence of a : after the period value indicates that it is approximate, a ? indicates that it is dubious.

The references for the Catalogue are given at the end, together with those of the notes; the source catalogue is also indicated according to the above code.

\section{The notes}

The notes are referred by means of the Rns number, e.g. the running number in Renson et al. (1991)'s General Catalogue of $A p$ and Am Stars.

Most of the notes are reported to indicate the binarity character of some stars.

Visual doubles are indicated if the companion star is closer than about 30 arcsec and the magnitude difference $\Delta m$ is smaller than 5 or 6 magnitudes. This because our aim is to evidentiate the possibility that the presence of a companion might jeopardize the measurements. The informations we give generally concern the ADS number (Aitken 1932), if available, the angular distance in arcsec, and the magnitude difference between the components.

The spectroscopic binary character is indicated if the period is known; these data are mainly from Batten et al. (1978) catalogue.

Some notes are introduced to point out errors found in the literature or to put in evidence some very interesting characteristic.

Acknowledgements. We would like to thank all colleagues who have supplied their results making easier the hard task of updating the catalogue.

\section{References}

Aitken R.G., 1932, New General Catalogue of Double Stars. Publ. Carnegie Inst. Washington No. 417

Batten A.H., Fletcher J.M., Mann P.J., 1978, Publ. Dominion Ap. Obs. 15, 121

Boss B., 1937, General Catalogue of 33342 Stars for the Epoch 1950.0, Publ. Carnegie Inst. Washington No. 468

Catalano F.A., Renson P., 1984, A\&AS 55, 371

Catalano F.A., Renson P., 1988, A\&AS 72, 1

Catalano F.A., Renson P., Leone F., 1991, A\&AS 87, 59

Catalano F.A., Renson P., Leone F., 1993, A\&AS 98, 269

Catalano F.A., Renson P., 1997, A\&AS 121, 57

Preston G.W., 1974, ARA\&A 12, 257

Renson P., Gerbaldi M., Catalano F.A., 1991, A\&AS 89, 429 